\title{
Antidiabetikum mildert Folgen einer Hirnschwellung
}

\author{
In einer Phase-II-Studie reduzierte der Sulfonylharn- \\ stoff Glibenclamid die ödembedingte Sterberate \\ nach einem Schlaganfall drastisch.
}

Nach Schätzungen kommt es bei $10-15 \%$ aller ischämischen Schlaganfälle zu gravierenden Hirnschwellungen, häufig sorgt hier nur eine dekompressive Kraniektomie für Erleichterung, die Sterberate liegt in solchen Fällen dennoch bei $40-60 \%$. Medikamentöse Ansätze sind rar und konnten bislang kaum überzeugen. Aufhorchen lassen daher die Ergebnisse einer Phase-IIStudie: Hier wurde zwar der primäre Endpunkt - eine Reduktion der nötigen chirurgischen Dekompressionen und ein gutes funktionelles Ergebnis - mit dem geprüften Sulfonylharnstoff Glibenclamid nicht erreicht, aber die ödembedingte Sterberate ließ sich signifikant senken. Sulfonylharnstoff-Rezeptoren sind auch bei der Entstehung von Hirnödemen nach einem Schlaganfall von Bedeutung, erläuterte Dr. Kevin Shet von der Yale University School of Medicine in New Haven. In Tierversuchen ließen sich mit solchen Substanzen Hirnschwellungen reduzieren, zudem werden neuroprotektive Mechanismen vermutet. In der Phase-II-Studie GAMES-RT prüften die Forscher um Shet die Gabe von Glibenclamid bei 83 Patienten mit einem großen ischämischen Hirninfarkt. Voraussetzung war ein Läsionsvolumen von mindestens 82 bis maximal 300 Kubikzentimeter im diffusionsgewichteten MRT. In Studien hätten sich 82 Kubikzentimeter als kritische Grenze für die Entwicklung eines malignen Infarkts erwiesen, so Shet. Patienten mit Thrombektomie wurden ausgeschlossen. Die Hälfte der Patienten erhielt innerhalb von zehn Stunden zusätzlich zur üblichen Therapie Glibenclamid, die übrigen Placebo. Die Präparate wurden per Bolus zum Therapiebeginn und mit anschließender Infusionen über 72 Stunden verabreicht. Als primären Endpunkt wählten die Forscher eine Kombination von Kraniektomierate und mRSWert von 0-4 Punkten nach 90 Tagen. Die Patienten waren im Schnitt 60 Jahre alt, hatten ein Läsionsvolumen von im Mittel 160 Kubikzentimeter und wurden rund neun Stunden nach Symptombeginn mit Glibenclamid behandelt.

\section{Vor allem jüngere Patienten profitieren}

Ohne Kraniektomie und mit einem mRS-Wert von 0-4 Punkten überstanden $42 \%$ mit Glibenclamid und 39\% der Patienten mit Placebo den Infarkt - der Unterschied war nicht signifikant. Auch der mRS-Wert allein betrachtet, war unter Glibenclamid tendenziell aber nicht signifikant besser (61\% vs. $47 \%$ ). Ebenso verfehlte die Sterberate nach 90 Tagen (17\% vs. $36 \%$ ) das Signifikanzniveau. Statistisch belastbare Vorteile für Glibenclamid gab es jedoch bei der Rate neurologisch bedingter Todesfälle (7\% vs. $25 \%)$ und vor allem bei der ödembedingten Sterberate (2\% vs. $22 \%$ ). Mit Glibenclamid wurden folglich kaum noch Todesfälle infolge von Hirnschwellungen beobachtet. Von der Therapie schienen vor allem jüngere Patienten zu profitieren: Bei den unter 70-Jährigen erreichten signifikant mehr mit dem Medikament als mit Placebo einen mRS-Wert von $0-4$ (69\% vs. $50 \%$ ). Schwere unerwünschte Effekte wurden unter Glibenclamid etwas seltener beobachtet als mit Placebo (68\% vs. $72 \%$; n. s.). Glibenclamid soll nun in einer Phase-III-Studie weiter untersucht werden.

Thomas Müller, Springer Medizin

Scientific Session 7, Stroke Clinical Trials: S7.004, GAMES-RT, a phase II study toward preventing edema after ischemia. 68. AAN-Kongress, Vancouver, 15. -21.4 .2016$

\section{Mitochondrientherapie stärkt Retinafunktion bei Parkinson}

\author{
Ein neues Medikament, das derzeit gegen erblich \\ bedingte Mitochondrienkrankheiten geprüft wird, \\ könnte auch bei Morbus Parkinson helfen. Darauf \\ deutet eine erste kleine Pilotstudie.
}

Bei der Entstehung von Morbus Parkinson scheinen oxidativer Stress und Störungen der mitochondrialen Atmungskette von Bedeutung zu sein. Diese Störungen, so die Hypothese, führen über die vermehrte Bildung freier Sauerstoffradikale zur Neurodegeneration. Versuche, diesen Prozess mit Coenzym Q und Vitamin E zu bremsen, sind jedoch in klinischen Studien gescheitert: Die Parkinson-Krankheit schritt damit trotzdem voran. Nun wagen Forscher einen neuen Versuch: Dr. Theresa Zesiewicz von der Universität in Tampa, Florida, präsentierte erste Ergebnisse einer kleinen Studie mit dem Wirkstoff EPI-743 (Alpha-Tocotrienol-Chinon). Die Substanz zielt auf Oxidoreduktasen - Enzyme, die Redoxreaktionen katalysieren. EPI743 soll vor sekundärem oxidativem Stress aufgrund einer
Glutathiondepletion schützen. Der Wirkstoff wird derzeit bei einigen vererbbaren Mitochondrienkrankheiten klinisch geprüft, unter anderem bei der Leber'schen hereditären Optikusneuropathie. Hier, so Zesiewicz, ließen sich in einer offenen Studie Ganglionzellen in der Retina vor freien Radikalen schützen. Auch beim Leigh-Syndrom, einer subakuten nekrotisierenden Enzephalomyelopathie, deutete eine erste Studie auf klinische Verbesserungen sowie erhöhte Spiegel von schützendem Glutathion.

\section{PERG-Amplitude und -Frequenz verbessert}

Effekte einer mitochondrialen Therapie lassen sich möglicherweise am leichtesten am optischen System nachweisen, so die Neurologin. Sie untersuchte, ob sich mit der Substanz visuelle Parameter bei Parkinson-Patienten verbessern lassen. Bei ihnen kommt es nicht selten zu Störungen bei der Farbwahrnehmung, Leseproblemen und trockenen Augen. Zehn ParkinsonPatienten erhielten sechs Monate lang $200 \mathrm{mg}$ oder $400 \mathrm{mg}$ des Wirkstoffs dreimal täglich. Neben der Retinafunktion - gemes- 